\title{
Differential Diagnosis and Management of Chronic Pericarditis in the Context of 2015 ESC Guidelines on Pericardial Diseases
}

\author{
Arsen D. Ristić, Dejan S. Simeunović, Petar Đukić, Mladen Kočica, Vesna Božić, Bosiljka \\ Vujisić-Tešić, Ivan Milinković, Ivana Živković, Vladimir Kanjuh, Petar M. Seferović. \\ Department of Cardiology, Department of Cardiac Surgery and Department of Pathology, Clinical Centre of Serbia and \\ Belgrade University School of Medicine, Belgrade, Serbia
}

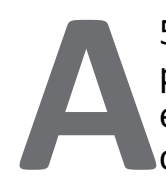

52 years old patient was admitted to our hospital because of the recurrent large pericardial effusion, only four weeks after previous pericardiocentesis and drainage of $1600 \mathrm{ml}$ of serous, initially idiopathic pericardial effusion. Five months before, he felt slowly progressing fatigue, dyspnea on effort and productive cough (but no hemoptysis). ECG revealed atrial fibrillation with low voltage and tachycardia and echocardiography demonstrated signs of an imminent cardiac tamponade and a foreign mass on the visceral layer of the pericardium in front of the right ventricle. Computed tomography verified epicardial infiltration of benign features in front of the right ventricle. The patient was initially stable after the first pericardiocentesis. However, soon after the hospital discharge, a large pericardial effusion slowly recurred, followed with dyspnea on effort. His medical history also includes arterial hypertension, well treated with ACE inhibitors.

Physical examination on his second admission revealed no fever, distended neck veins, and distant heart sounds. His heart rate was rapid but now regular (110 b/min, no atrial fibrillation any more) and there was no severe hypotension (BP 120/80 $\mathrm{mmHg}$ ). In addition to the rapid heart rate, ECG revealed low voltage and electrical alternans, with a leftward axis deviation (QRS vector $-26^{\circ}$ ). After repeated pericardiocentesis his heart rate returned to $70-80 / \mathrm{min}$. However, a brief paroxysm of atrial fibrillation with a rapid ventricular rate of $180 \mathrm{~b} / \mathrm{min}$ occurred again immediately after pericardiocentesis.

Laboratory analyses revealed increased sedimentation rate during the course of hospitalization (46... 38... $80 \ldots 50 \ldots 100 \ldots 69)$ with a slight syderopenic anemia (hemoglobin 111-122, serum iron 7.3). All other routine laboratory analyses were normal including serum cholesterol 4.19, HDL 1.41, LDL 2.22 and triglycerides. Although other parameters of the liver function were normal, gamma GT was significantly increased (132) probably due to the previous alcohol abuse.

Chest X-ray demonstrated small pleural effusion at the left side and an enlarged "water-bottle" heart shadow. Echocardiography revealed a very large, Horowitz type $D$ pericardial effusion with the diastolic separation of pericardial layers of $24-30 \mathrm{~mm}$ in front of the right ventricle and 33-36 mm behind the left ventricle. Interestingly, there was a 15-20 mm thick, pear-like formation at the visceral pericardium in front of the right ventricle. Despite the huge size of the effusion, there were no signs of cardiac tamponade. Remaining echocardiography findings were normal, with the normal size and contractility of all cardiac structures.

Abdominal ultrasonography has shown normal size and shape of the liver with no focal lesions, but diffusely non-homogenous structure. Portal vein was normal, with no thrombosis. Gall bladder was of normal size and position, but bended, with increased wall thickness and with an infundibular content of high density but no calculosis. Choledochus was of regular luminal size, with no calculosis. Pancreas was normal, as well as the spleen and the kidneys. No ascites could be detected.

As a part of the initial management, pericardiocentesis and drainage of $1700 \mathrm{ml}$ of hemorrhagic pericardial effusion was performed using fluoroscopic control in the cardiac catheterization laboratory, a flexible 7F catheter and the subxiphoid approach. Pericardial fluid analyses revealed the following findings: glucose 2.6, total proteins 44, albumins 27, alpha amylase 37, LDH 745 U/l, pH 8. All cultures for aerobic and anaerobic bacteria remained sterile. Direct microscopy and cultures for tuberculosis were negative as well. Cytological examination of the pericardial effusion has demonstrated coagulated proteins, scarce lymphocytes, granulocytes, and rare solitary cells with week expression of anisomorphism.

Cardiac catheterization was performed in the second session, in order to exclude presence of any pathological vascularisation of the timorous formation on the visceral layer of the pericardium in front of the right ventricle. However, coronary arteries were normal, as well as the left ventricle with the LVEF of $65 \%$. Right-heart catheterization revealed normal pressures in the pulmonary circulation and the right-heart chambers.

In order to additionally visualize the tumor formation, intracardiac echocardiography was performed (Accu-Nav, 
Siemens, Germany). Scanning from the right atrium, it was possible to visualize pear-like tumor on the visceral pericardium in front of the right ventricle, $3 \times 5 \mathrm{~cm}$, arising from the epicardium on the wide basis, with distinct borders and no signs of infiltration of the myocardium. Small, thick, residual pericardial effusion was also detectable, despite the previous drainage of the effusion and the presence of a functional pericardial catheter.

In an attempt to clarify the etiological diagnosis, after 12 days of prolonged drainage of the pericardial effusion with a daily drainage of 200-300 ml of sero-hemorrhagic effusion, the patient underwent pericardioscopy and pericardial biopsy: (16.5 F Olympus HYF-1T flexible endoscope) (Figure 1). Endoscopic evaluation revealed signs of hemorrhagic inflammation on both pericardial layers. As expected according to the echocardiography and computed tomography findings, a large, solitary tumor formation, with macroscopic features of a lipoma, was visualized on the epicardial layer in front of the right ventricle. Six biopsy samples were taken from the borderline area of the tumor and surrounding epicardium and pericardium. The procedure was completed without any complications.

Patohistological examination has revealed lymphocyte infiltration and changes specific for cholesterol pericarditis as depicted in the Figure 1.

Due to the continuous production of large amounts of sero-hemorrhagic fluid the patient was referred to pericardiectomy. Subtotal pericardiectomy was performed, but surprisingly $5 \times 3 \mathrm{~cm}$ large tumor, previously

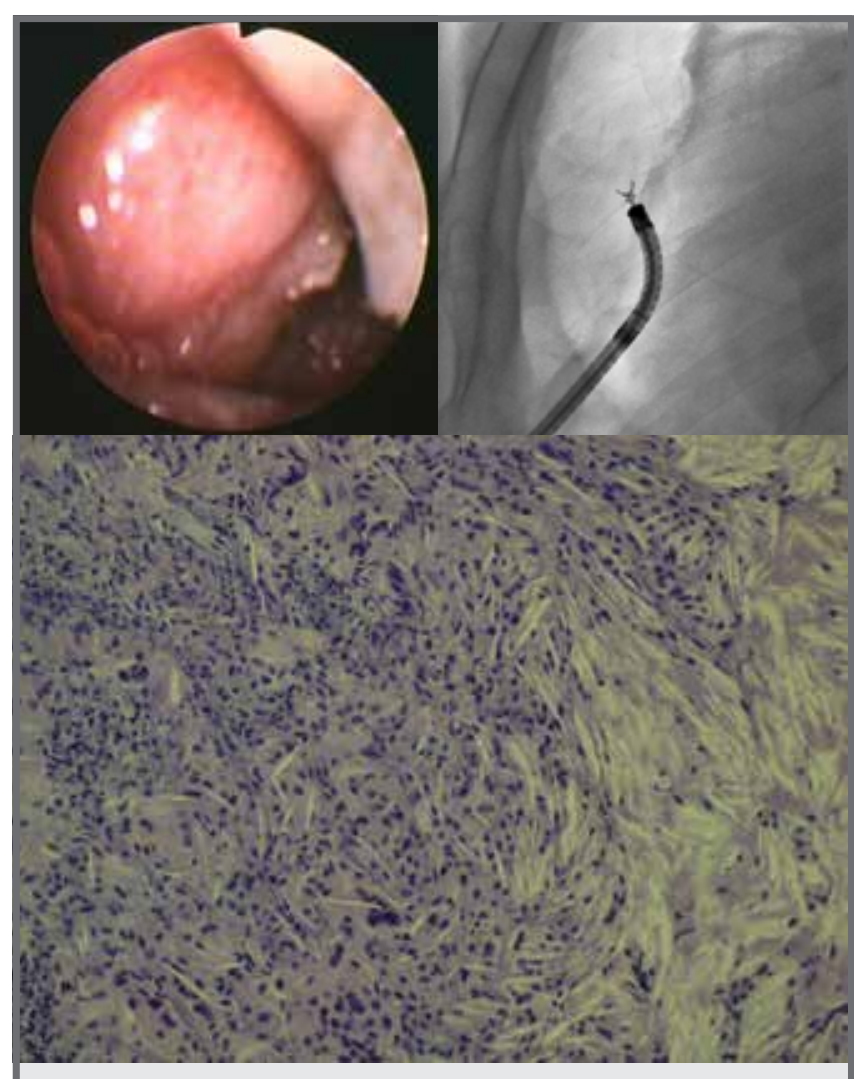

Figure 1. Pericardioscopy and pericardial biopsy in a patient with recurring cardiac tamponade and extensive prolonged production of sero-hemorrhagic pericardial effusion of unclear etiology. Patohistology revealed changes specific for cholesterol pericarditis. confirmed by echocardiography, computed tomography, intracardiac echocardiography, and pericardioscopy was not present at the time of surgery any longer. Perhaps, a lipomatous content of the tumor was drained after the biopsy. However, several similar tumors (Figure 2) were detected in the left pleura and removed during the same surgical procedure. In addition, $300 \mathrm{ml}$ of thick chylous effusion was evacuated from the left pleura. The final diagnosis established from the biopsy samples taken by pericardioscopy and the samples taken during the surgery was cholesterol pericarditis with the chronic inflammation (foreign body granulomatous inflammation around the cholesterol crystals) as well as the benign pleural lipoma. Pear-like infiltration of the visceral pericardium in front of the right ventricle was most probably also a lipoma which collapsed after taking several biopsy samples. After the surgery, there was no relapse of either pericardial or pleural effusion and the patient was discharged from the hospital on antihypertensive medication and statins. The patient remained stable, with no symptoms, no pericardial or pleural effusion and no recurrences of lipomatous tumors during the 10-years of follow-up. He was even able to return to his previous profession - acting in the classical drama theatre.

\section{Chronic pericarditis as a diagnostic and therapeutic challenge}

The most important prerequisite for successful management of patients with chronic pericarditis is determination of the background etiology. Therefore, extensive etiological search may be needed in order to uncover the specific disease affecting the pericardium, sometimes, including pericardial fluid analyses and targeted pericardial biopsies. Although indications for pericardiectomy are well-established in constrictive pericarditis, referral of patients with chronic pericardial effusion for surgery is still a matter of debate, despite the clear instructions from the ESC Guidelines [1-3] and the American Society of Echocardiography clinical recommendations for multimodality cardiovascular imaging of patients with pericardial disease [4].

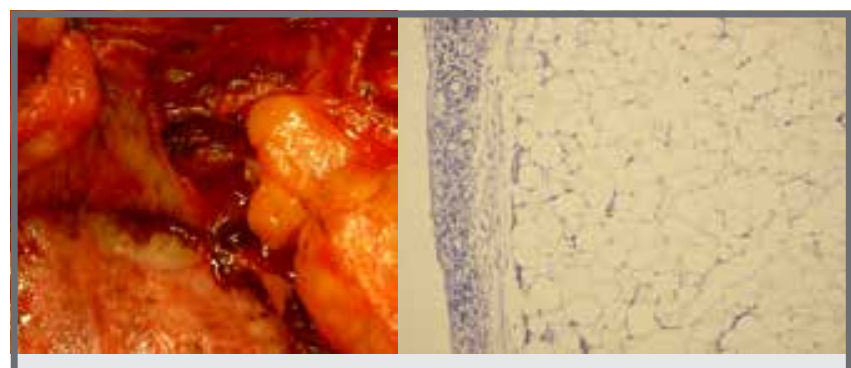

Figure 2. Intraoperative findings demonstrating extensive inflammatory changes on the visceral and parietal pericardium, thickened parietal pericardium and infiltration in the left pleural space (white arrow). Surprisingly, no timorous infiltration of the visceral pericardium could be visualized in front of the right ventricle any more. Right-sided image reveals histological examination from the pleural tumor - pleural lipoma. 


\section{Discussion}

When a pericardial effusion is detected, the first step is to assess its size, hemodynamic importance (especially the presence of cardiac tamponade) and possible associated diseases (either cardiovascular or systemic diseases). Pericardial effusion is often associated with known or unknown (e.g. hypothyroidism) medical conditions (up to $60 \%$ of cases). If inflammatory signs are present, the clinical management should be that of pericarditis. Cardiac tamponade without inflammatory signs is associated with a higher risk of a neoplastic etiology (likelihood ratio 2.9), whereas a severe effusion without cardiac tamponade and inflammatory signs is usually associated with a chronic idiopathic etiology (likelihood ratio 20$)^{1}$.

Therapy of pericardial effusion should be targeted at the etiology as much as possible. In about $60 \%$ of cases, the effusion is associated with a known disease and the essential treatment is that of the underlying disease. When a pericardial effusion becomes symptomatic without evidence of inflammation, drainage of the effusion should be considered since there are no proven effective medical therapies to reduce an isolated effusion. In the absence of inflammation, NSAIDs, colchicine and corticosteroids are generally not effective. The major specific causes to be ruled out are bacterial pericarditis, neoplastic infiltration and pericarditis associated with a systemic autoimmune or metabolic disease. Each of these specific causes has a frequency of $<5 \%$ of all unselected cases of pericarditis from developed countries while frequencies increase in moderate to large pericardial effusions. The etiological spectrum is different in developing countries with a high prevalence of tuberculosis (e.g. $70-80 \%$ of pericarditis in sub-Saharan Africa, and often associated with HIV infection). Rare patients with relapsing pericarditis can also benefit from pericardiectomy $y^{1-3}$.

Table 1. Algorithm for diagnostic evaluation of patients with chronic pericarditis according to the 2015 ESC Guidelines: First and second level investigations for pericarditis ${ }^{1}$.

\begin{tabular}{|l|l|}
\hline $\begin{array}{l}\text { Diagnostic } \\
\text { tools }\end{array}$ & Investigation \\
\hline $\begin{array}{l}1^{\text {st }} \text { level } \\
\text { methods }\end{array}$ & $\begin{array}{l}\text { Markers of inflammation (ESR, CRP, WBC). } \\
\text { Renal function and liver tests, thyroid function. } \\
\text { Markers of myocardial lesion (troponins, CK) } \\
\text { ECG } \\
\text { Echocardiography } \\
\text { Chest X-ray }\end{array}$ \\
\hline $\begin{array}{l}2^{\text {nd }} \text { level } \\
\text { methods }\end{array}$ & $\begin{array}{l}\text { CT and/or CMR, pericardiocentesis, or } \\
\text { surgical drainage, for (i) cardiac tamponade } \\
\text { or (ii) suspected bacterial, neoplastic } \\
\text { pericarditis, or (iii) symptomatic moderate to } \\
\text { large effusions not responding to aetiologies } \\
\text { according to clinical presentation (presence } \\
\text { of high risk clinical criteria). }\end{array}$ \\
\hline
\end{tabular}

CK - creatine kinase; CMR - cardiac magnetic resonance; CRP - C-reactive protein; CT - computed tomography; ECG - electrocardiogram; ESR -erythrocyte sedimentation rate.

Cholesterol pericarditis is a rare complication of chronic pericardial effusion or chronic scarring of the pericardium $^{5}$ and is exacerbated by cholesterol crystals. Common underlying causes include tuberculous pericarditis, autoimmune rheumatic diseases, and pericardial trauma [6, 7]. When a pericardial effusion is relatively acute, its cholesterol content remains in solution. However, when the pericardial effusion is chronic, the normal ability to dissolve cholesterol is impaired and cholesterol crystals are deposited in the pericardium and effusion ${ }^{8-10}$. The fluid is clear, in contrast to chylopericardium, and classically is said to have a glittering "gold paint" appearance, as it was the case with our patient at the first pericardiocente$\operatorname{sis}^{11,12}$. However, any other macroscopic appearance of pericardial effusion apart from the "gold paint" is not excluding the diagnosis.

The effusions tend to be large. The concentration of cholesterol equals or exceeds that of the blood, often attaining values above $500 \mathrm{mg} / \mathrm{dL}(13 \mathrm{mmol} / \mathrm{L})^{12,13}$. Unfortunately, estimation of cholesterol level was not a part of our routine evaluation of the pericardial effusion, which turned out to be wrong in this specific case. The pericardial effusion associated with myxedema also has a high cholesterol concentration, but crystals are usually absent.

Blood associated with inflammation is thought to be the source of cholesterol in the pericardial fluid, and evidence of current or previous hemorrhage is usually evident. The pericardium is thicker than normal (Figure 2 left) and its inner surface is lined with plaques and cholesterol deposits. The histological findings include fibrosis, inflammatory cells, cholesterol clefts and crystals of variable geometry, and giant cell granulomata.

Treatment includes pericardiocentesis, which is seldom effective over the long-term because the effusions tend to recur and can cause tamponade at any time ${ }^{13}$. This procedure also fails to address the thick, scarred pericardium and does not prevent the late development of constrictive pericarditis. Thus, optimal therapy is radical pericardiectomy with additional treatment of the underlying cause of chronic pericarditis ${ }^{1-3}$.

\section{Conclusion}

Differential diagnosis may be complex in patients with chronic pericarditis and according to both 2015 ESC guidelines and American Society of Echocardiography clinical recommendations require systematic application of multimodality imaging including targeted pericardial biopsies. In patients poorly responding to medical treatment or with specific etiology highly prone to constriction, pericardiectomy should be also considered as the ultimate treatment option.

\section{References}

1. Adler $Y$, Charron $P$, Imazio $M$, Badano L, Barón-Esquivias $G$, Bogaert J, Brucato A, Gueret P, Klingel K, Lionis C, Maisch B, Mayosi B, Pavie A, Ristić AD, Sabaté Tenas M, Seferovic P, Swedberg K, Tomkowski W, Achenbach S, Agewall S, Al-Attar N, Angel Ferrer J, Arad M, Asteggiano R, Bueno H, Caforio AL, Carerj S, Ceconi C, Evangelista A, Flachskampf F, Giannakoulas G, Gielen S, Habib G, Kolh P, Lambrinou E, Lancellotti P, Lazaros G, Linhart A, Meurin P, Nieman K, Piepoli MF, Price S, Roos-Hesselink J, Roubille F, Ruschitzka F, Sagristà Sauleda J, Sousa-Uva M, Uwe Voigt J, Luis Zamorano J; European Society of Cardiology (ESC). 2015 ESC Guidelines for the diagnosis and management of pericardial diseases: The Task Force for the Diagnosis and Management of Pericardial Diseases of the 
European Society of Cardiology (ESC) Endorsed by: The European Association for Cardio-Thoracic Surgery (EACTS). Eur Heart J 2015;36(42):2921-64.

2. Maisch B, Seferović $P M$, Ristić AD, Erbel R, Rienmüller R, Adler $Y$, Tomkowski W, Thiene G, Yacoub MH. European Society of Cardiology Guidelines: Diagnosis and management of the pericardial diseases. Executive summary. Eur Heart J 2004;25(7):587-610.

3. Cho YH, Schaff HV. Surgery for pericardial disease. Heart Fail Rev 2013;18(3):375-87.

4. Klein AL, Abbara S, Agler DA, Appleton CP, Asher CR, Hoit B, Hung J, Garcia MJ, Kronzon I, Oh JK, Rodriguez ER, Schaff HV, Schoenhagen $P$, Tan CD, White RD. American Society of Echocardiography clinical recommendations for multimodality cardiovascular imaging of patients with pericardial disease: endorsed by the Society for Cardiovascular Magnetic Resonance and Society of Cardiovascular Computed Tomography. J Am Soc Echocardiogr 2013;26(9):965-1012.

5. Maisch $B$, Ristić AD. Diagnostics and therapy of pericarditis and pericardial effusion. Herz 2014;39(7):837-56.

6. Cosyns B, Plein S, Nihoyanopoulos P, Smiseth O, Achenbach S, Andrade MJ, Pepi M, Ristic A, Imazio M, Paelinck B, Lancellotti P; European Association of Cardiovascular Imaging (EACVI); European Society of Cardiology Working Group (ESC WG) on Myocardial and Pericardial diseases. European Association of Cardiovascular Imaging (EACVI) position paper: Multimodality imaging in pericardial disease. Eur Heart J Cardiovasc Imaging 2015;16(1):12-31.
7. Pankuweit S, Ristić AD, Seferović PM, Maisch B. Bacterial pericarditis: diagnosis and management. Am J Cardiovasc Drugs 2005:5(2):103-12.

8. Raposo L, Andrade MJ, Ferreira J, Aguiar C, Abecasis M, Ramos $S$, Reis AB, Martins AP, Pais MJ, Gomes RS. Cholesterol pericarditis--relapsing pericardial effusion in a patient with rheumatoid arthritis. Rev Port Cardiol 2005;24(5):737-45.

9. Ford EJ, Bear PA, Adams RW. Cholesterol pericarditis causing cardiac tamponade. Am Heart J 1991;122(3 Pt 1):877-9.

10. Noiri E, Itoh T. Disorder of pericardial fluid cholesterol metabolism in cholesterol pericarditis. Jpn J Med 1991;30(4):393.

11. Maisch B, Ristić AD, Seferović PM, Tsang TSM. Interventional Pericardiology: Pericardiocentesis, Pericardioscopy, Pericardial Biopsy, Balloon Pericardiotomy, and Intrapericardial Therapy (DVD incl). Springer Verlag, Heidelberg 2011

12. Brawley, RK, Vasko, JS, Morrow, AG. Cholesterol pericarditis. Considerations of its pathogenesis and treatment. Am J Med 1966; 41:235.

13. Ristić $A D$, Imazio $M$, Adler $Y$, Anastasakis $A$, Badano LP, Brucato $A$, Caforio AL, Dubourg O, Elliott P, Gimeno J, Helio T, Klingel K, Linhart A, Maisch B, Mayosi B, Mogensen J, Pinto Y, Seggewiss H, Seferović PM, Tavazzi L, Tomkowski W, Charron P. Triage strategy for urgent management of cardiac tamponade: a position statement of the European Society of Cardiology Working Group on Myocardial and Pericardial Diseases. Eur Heart J 2014;35(34):2279-84. 